\title{
Finding the depth of radioactivity in construction materials
}

Malcolm J. Joyce BSc, PhD, DEng, CPhys, CEng

Head of Department, Department of Engineering, Lancaster University, Lancaster, UK

Jamie C. Adams BSc, PhD

Researcher, Department of Engineering, Lancaster University, Lancaster, UK
John A. Heathcote MA, PhD, FGS, CGeol

Principal Scientist, Dounreay Site Restoration Ltd (DSRL), Dounreay, Thurso, UK

Matthew Mellor MA, DPhil

Director, Createc Ltd, Derwent Mills Commercial Park, Cockermouth, UK

\begin{abstract}
A key challenge in disposing of nuclear legacy facilities and planning a new nuclear plant is how to assess the extent or likelihood of radioactive contamination in construction materials and the ground. This paper summarises the status of two techniques based on the analysis of emitted radiation from materials that comprise such structures, and describes how this analysis can be used to infer the depth of contamination without the need to penetrate the structure or to destroy it in the process. Two experimental facilities have been developed to test the efficacy of these techniques, and data are provided for the most widespread contaminant experienced in the sector: caesium-137. Finally, the influence on the technique of the likely variety of silica-based media to be encountered in the nuclear industry is described, together with a summary of challenges to be addressed in future research.
\end{abstract}

\section{Notation \\ $d \quad$ depth in the material \\ E photon energy \\ $I(x) \quad$ intensity of the radiation \\ $I(0) \quad$ intensity at $x=0$ \\ $R_{0}$ and $R_{\mathrm{X} / \gamma}$ ratios of the counts in the X-ray and $\gamma$-ray photopeaks at zero depth and depth $d$ \\ $x$ $x$ and $y \quad$ constants and $\varphi$ is a function of an angle $\theta$ derived between successive principal components derived at increasing depths $\mu(E) \quad$ attenuation factor $\mu_{\mathrm{X}}$ and $\mu_{\gamma}$ attenuation coefficients for the X-ray and the $\gamma$-ray, respectively}

\section{Introduction}

Although the fission process results in a plethora of radionuclides as byproducts, one of the silver linings in this cloud of potentially harmful radioactive contamination is that the vast majority of these isotopes decay rapidly to the point at which they pose no harm. Of those that remain, there are two in particular that pose a risk, justifying long-term care and maintenance, and which are amenable to the technique described in this work: caesium-137 $\left({ }^{137} \mathrm{Cs}\right)$ and isotopes of plutonium, and, to a lesser, extent cobalt-60 ( $\left.{ }^{60} \mathrm{Co}\right) .{ }^{137} \mathrm{Cs}$ arises as a fission product, while plutonium arises as a byproduct of neutron capture on uranium, and both can be transported extensively by the ingress of water. The latter was processed extensively for military purposes in the early days of the nuclear industry. ${ }^{60} \mathrm{Co}$ can arise as a result of the neutron activation of steels in built structures, especially steels high in cobalt where they are used in specialist nuclear applications. As a result of spills and leaks in buildings and the surrounding ground, these substances can pose a restriction on the ease with which structures and land are decommissioned. They have halflives that are sufficiently long that radioactive decay may not be acceptable as the method of dealing with materials contaminated by them. As a result, these substances can constitute the majority of contaminated structural materials that have to be characterised and disposed of in dealing with the UK nuclear legacy.

While the nuclear properties of these radionuclides are well understood, it is largely their chemistry that dictates their behaviour in the natural environment. As such, cobalt can be dispersed by transport in which contaminated high-cobalt steels contact with structural materials, while caesium readily forms water-soluble salts that migrate rapidly along with the egress of water, eventually becoming strongly bound with the concrete itself, or with mineral grains (particularly micas) in the soil. As a result, these contaminants are a significant problem when decommissioning spent nuclear fuel storage ponds at nuclear power plants, nuclear process plant, and evaporation pans under care and maintenance regimes. They are found in the form of localized particulate debris, such as fragments of fuel found occasionally on beaches around Dounreay, and in the form of dispersed distributions of radioactivity in porous media constituting the structure of buildings and the ground under and around them. Because the radiation is reasonably penetrating (fractions of a metre in soil and concrete), detection of the general presence of contamination is reasonably easy. However, the shielding effect of the medium in which it is 
entrained can disguise the extent of contamination and render accurate estimates of disposal amounts (and therefore costs and potential dose to workers) difficult to achieve before intrusion. Significant quantities of material require characterisation at Sellafield, such as an estimated 13 million $\mathrm{m}^{3}$ of soil potentially classed as nuclear waste (Cruickshank, 2012). The costs of disposal could be vast if a technique is not available for the non-destructive assay of such a legacy with which to support the separation of this waste stream according to the established categories for radioactive waste. While core drilling is in widespread use to identify the precise location of contamination (see, e.g., Fourie, 2012), this approach generates waste, risks the exposure of those workers carrying out the drilling and can also miss contamination adjacent to the site of the core extraction. It is also expensive, time consuming and logistically inconvenient, as can be appreciated from Figure 1.

This paper summarises the state of the art of a technique developed to estimate the depth of contamination in silicabased materials such as soils, sands, concrete and hardcore, non-destructively and non-intrusively. This technique is based on the influence that the contaminated material has on the

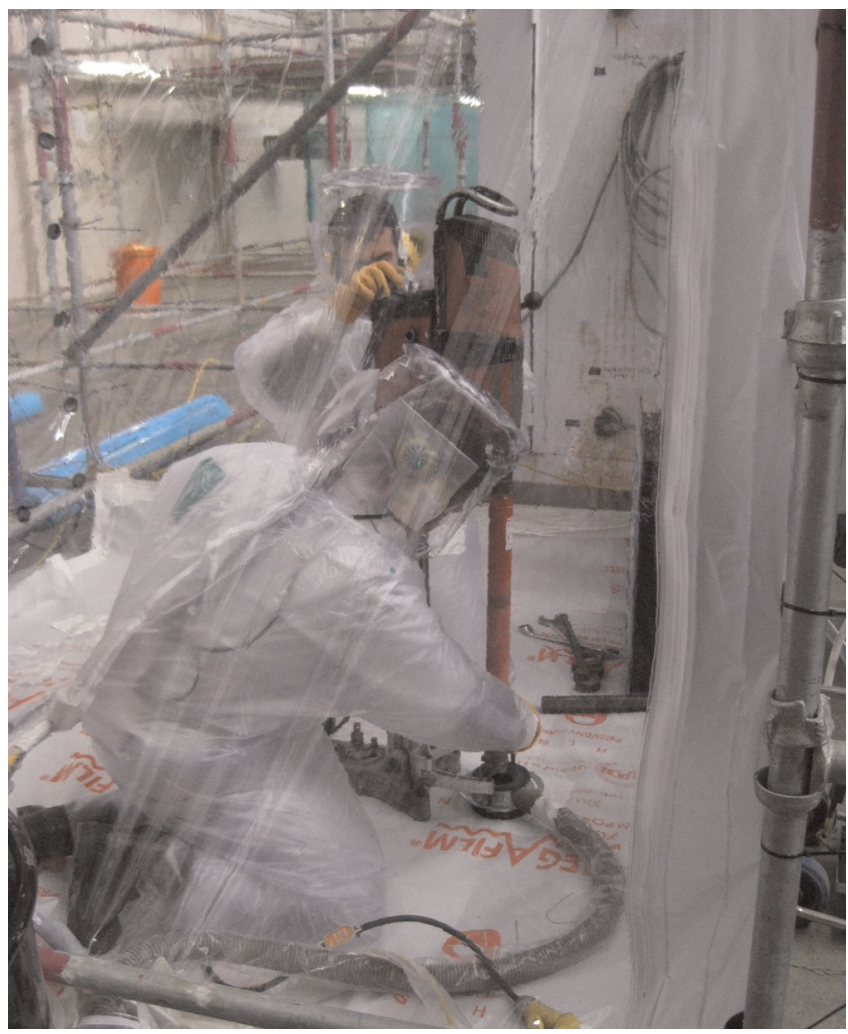

Figure 1. Two operatives undertaking a core-drilling procedure in a nuclear decommissioning environment, November 2009 (reproduced by permission of Dounreay Site Restoration Ltd and the Nuclear Decommissioning Authority) radiation emitted by the contamination. This paper will focus on its use with respect to ${ }^{137} \mathrm{Cs}$.

\section{Supporting theory and proof-of-principle measurements}

All materials to a greater or lesser extent act to attenuate the transmission of electromagnetic radiation through them, as a result of the interaction of this radiation with the electronic shell structure of the atoms comprising the material. This phenomenon is highly dependent on the number of electrons present, that is the atomic number $Z$ of the specific atom with which the interaction is taking place, with high $Z$ metals generally being the most effective at attenuating X-rays and $\gamma$-rays in the context of this work.

The degree of attenuation is also dependent on the energy of the radiation, with low-energy photons being more readily attenuated than their high-energy counterparts. This phenomenon has long been approximated by an exponential relationship of the form

1. $I(x)=I(0) e^{-\mu(E) x}$

where $I(x)$ is the intensity of the radiation at a thickness $x$ of material, $I(0)$ is the intensity at $x=0, \mu(E)$ is the attenuation factor for that material as a function of energy $E$.

Almost all radionuclides that emit photons in the $\gamma$-ray region of the electromagnetic spectrum emit a sequence of several photons of different energies as a result of the decay cascade of quantum states in the nucleus. As a result they exhibit a characteristic $\gamma$-ray spectrum that extends typically from a few $\mathrm{keV}$ to a few $\mathrm{MeV}$. Therefore, photons from the same nucleus are attenuated differently, based entirely on the difference in the energies of their photon emissions. In this respect, ${ }^{137} \mathrm{Cs}$ is an excellent example because it (or specifically its short-lived meta-stable daughter product, barium-137m $\left({ }^{137 m} \mathrm{Ba}\right)$ ) emits a characteristic $\gamma$ ray at $662 \mathrm{keV}$ (and a very weak decay at $284 \mathrm{keV}$, which is usually neglected) together with a collection of X-ray emissions at approximately $37 \mathrm{keV}$. When attenuated by contrasting thicknesses of materials, corresponding changes to the photon spectra as depicted in Figure 2 are observed. In Figure 2, data are presented arising from exploratory measurements with a cadmium telluride detector (XRF Corp., Woburn, MA, USA) and a sealed point ${ }^{137} \mathrm{Cs}$ source placed at two comparison depths in a bespoke, sand-filled phantom (Shippen and Joyce, 2011). The term 'phantom' is more often used in medical radiotherapy contexts when it is desirable to replicate the effect of living tissue on the attenuation of radiation. In the current context, the role of the phantom is to replicate a small section of the concrete medium under scrutiny without the need to produce large amounts of radioactively contaminated material. Superior energy resolution can be achieved with hyper-pure 


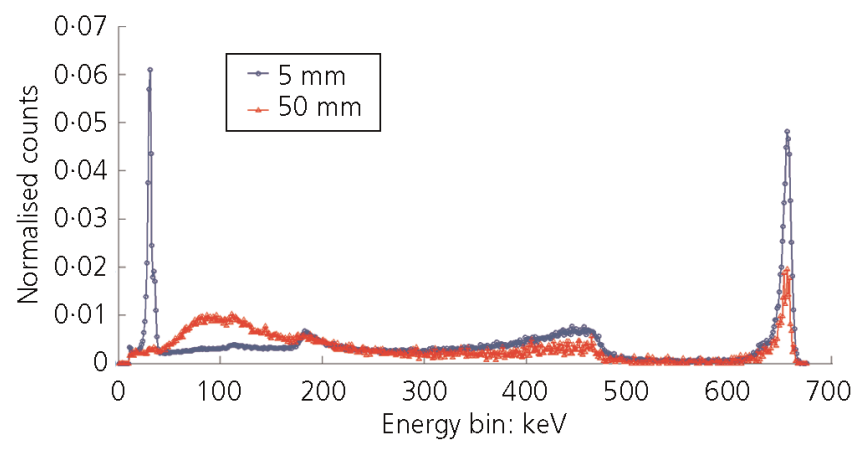

Figure 2. Two spectra for a sealed, point caesium-137 source inserted at depths $5 \mathrm{~mm}$ (circles) and $50 \mathrm{~mm}$ (triangles) in a silica-sand filled phantom. The X-ray photopeak at $5 \mathrm{~mm}$ (circles trace, left) is absent at $50 \mathrm{~mm}$, while the 662-keV photon (both traces, right) is present at both depths, but significantly attenuated at $50 \mathrm{~mm}$ compared with $5 \mathrm{~mm}$ by approximately 50\% (Adams et al., 2011)

germanium detectors as opposed to cadmium telluride if that is desired (see, e.g., Mahe, 2011); however, these are often expensive and require cryogenic cooling. They are not essential in the method summarised in this paper. The phantom is shown in Figure 3 . The effect of varying the depth of the source between the extremes of shallow $(5 \mathrm{~mm})$ and deep $(50 \mathrm{~mm})$ is observed qualitatively in the energy-dependent attenuation of the lowenergy photon peak at approximately $37 \mathrm{keV}$, relative to the reduced degree of attenuation that is characteristic of the higher energy photopeak at $662 \mathrm{keV}$.

\section{Commissioning quantitative measurements}

In order to extract quantitative information about the depth of contamination based on this phenomenon, a relationship between the characteristic photon decays in ${ }^{137} \mathrm{Cs}$ has been

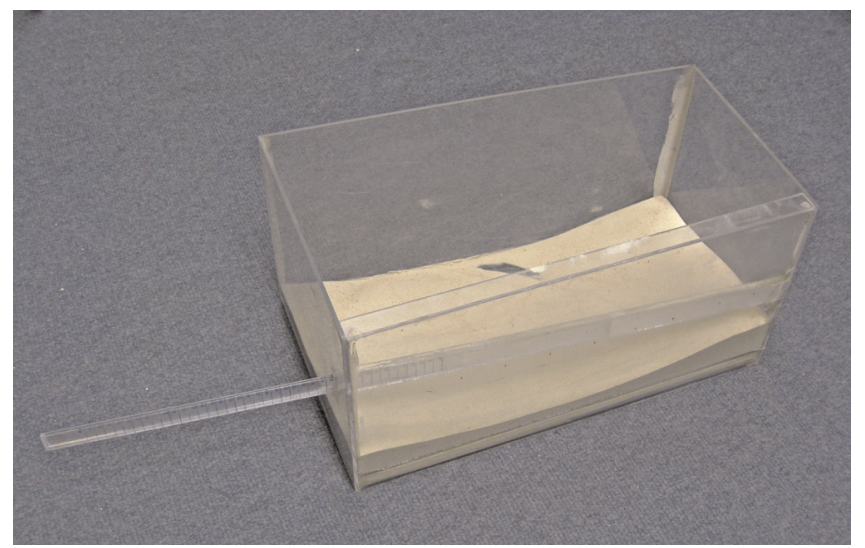

Figure 3. A photograph of the sand-filled phantom designed and commissioned to support this research, with most of the sand removed to expose the calibrated slider (Adams et al., 2011; Shippen and Joyce, 2011) derived (Shippen and Joyce, 2010). Given that the decay of both the $\mathrm{X}$ ray and $\gamma$ ray conform to the relationship of Equation 1, these two expressions can be combined as a simultaneous pair of equations to give an expression for depth, $d$

2. $\quad d=\frac{1}{\mu_{\mathrm{X}}-\mu_{\gamma}} \log _{e} \frac{R_{0}}{R_{\mathrm{X} / \gamma}}$

where $\mu_{\mathrm{X}}$ and $\mu_{\gamma}$ are the attenuation coefficients for the $\mathrm{X}$ ray and the $\gamma$ ray, respectively, and $R_{0}$ and $R_{\mathrm{X} / \gamma}$ are the ratios of the counts in the X-ray and $\gamma$-ray photopeaks at zero depth and depth $d$ respectively.

Using this expression and a campaign of related measurements with the silica-sand phantom referred to above, it is possible to extract the linear data supporting the expression in Equation 2 and thus to estimate the limiting uncertainty for the method in the case of sealed, point sources of the type that might replicate insoluble particulate matter. Data of this type are provided in Figure 4 (Shippen and Joyce, 2010, 2011), where a plot of $\log _{e} R_{0} / R_{\mathrm{X} / \gamma}$ against depth $d$ is given for the case of silica sand along a linear fit to the data. Estimates of uncertainty associated with the inference of depth on the basis of this calibration data indicate depth estimates can be made to within $\pm 1 \mathrm{~mm}$.

Returning to Figure 2, it is clear that in addition to the influence on the photopeaks that represent the low and high energy limiting features in this spectrum, a further influence of the attenuation by the material in which the contamination is entrained is observed on the continuum of data between photon energies of approximately $50 \mathrm{keV}$ and $500 \mathrm{keV}$. The photons contributing to this region, commonly referred to as the Compton background, arise as a result of photons that interact only partially in the detector (scattering back out into

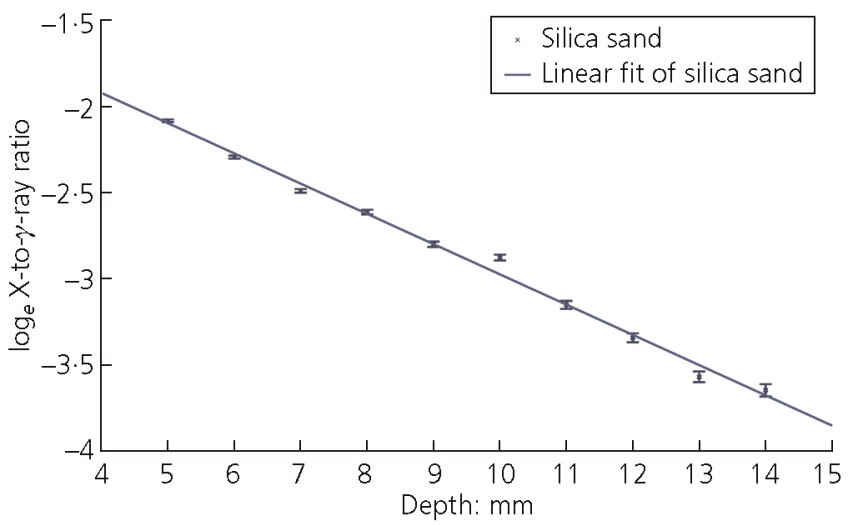

Figure 4. A plot of $\log _{e} R_{0} / R_{X / \gamma}$ against depth $d$ for a sealed, point source entrained in dry silica sand together with a linear fit to the data (Shippen and Joyce, 2010, 2011) 


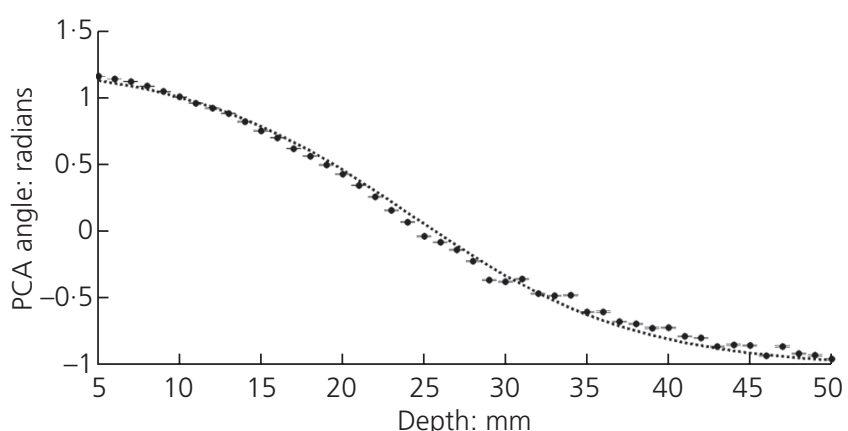

Figure 5. A plot of the principal component analysis (PCA) angle $\theta$ between the two lowest-order principal components identified for a selection of $\gamma$-ray spectra recorded for successive depths $d$ for silica sand, together with a non-linear fit of Equation 3 (Adams et al., 2011)

the environment) and also those photons that scatter within the material in which the contamination is entrained, losing some of their energy before being detected. The phenomenon is clearly related to depth, but is a much more complicated dependence than the linear dependence associated with the photopeaks. To exploit the additional information that exists as a result of this, principal component analysis (PCA) has been used to highlight the two lowest-order components of this dependence. When these are plotted as a function of depth, a dependence is observed that can be used as a calibration curve in a similar way to the approach depicted in Figure 4 (Adams et al., 2011). This is shown in Figure 5 for the case of ${ }^{137}$ Cs.

PCA is a long-established analytical method to extract salient patterns in multivariable datasets. The fundamental approach encompassed by PCA is derived from the covariance of the dataset. For example, a dataset of $n$ variables, a $n \times n$ covariance matrix can be derived from which the corresponding eigenvectors can be extracted (numerically for the case of a large number of variables). When ordered in terms of eigenvalue, the resulting list provides a rank of the components describing the data in order of significance, that is which describe the most salient trends in the data. For a simple, two-dimensional $x, y$ dataset, in which all the data follow a linear trend without any additional variance, only one component corresponding to the eigenvector describing the linear dependence results. Alternatively, if such a dataset did exhibit variance in addition to the linear trend (i.e. some data offset from the line in a direction orthogonal to the first component) this variance would be described by higher orders of components orthogonal to the first component. In practice, the number of components describing a dataset is usually limited to the first two or three as these provide sufficient insight for most applications. If desired, the original data can then be extracted back that accord with these components, in which case the dataset is effectively refined in terms of the selected components with the higher-order variances removed. Therefore, for a complex, dispersed set of data in $x, y$ the returned data under PCA limited to two components would describe two orthogonal lines that highlight the two principal trends in the data, excluding all higher-order trends. For the data in this work the two most significant principal components have been extracted for each depth calibration and a continuous dependence between these components with depth has been extracted.

An empirical fit to the data yields a general, non-linear relationship for all entrained, scatter photon data analysed in terms of principal components

3. $d(\varphi)=x+y \log _{e} \varphi$

where $d$ is depth, $x$ and $y$ are constants and $\varphi$ is a function of an angle $\theta$ derived between successive principal components derived at increasing depths. The specific advantage of this approach over the approach described earlier that is based on Equation 2 is that, in principle, any $\gamma$-ray spectra subject to the effects of scattering as a result of being entrained at some depth in a material can be calibrated to derive the parameters $x, y$ and $\varphi$, and thus an estimate of depth can be extracted. This approach has been subject to blind tests in which the previous depth of sealed source locations were not known, in which it performed very well estimating the depths of the source radioactivity to within an average of $2 \mathrm{~mm}$ uncertainty down to depths of $45 \mathrm{~mm}$ (Adams et al., 2011).

The technique based on Equation 2, although simpler to derive in absolute terms, is dependent on the radionuclide in question having sufficiently prominent photopeaks with a sufficient separation in energy to yield a contrast in terms of their attenuation, which is often not the case. Compton scattering is a process that always occurs. For the cases of ${ }^{60} \mathrm{Co}$ and plutonium, in particular, many fewer features exist to provide a basis on which the photopeak approach can be exploited and thus the PCA method has much more potential for these nuclides. Because the $37 \mathrm{keV} \mathrm{X}$ ray from ${ }^{137 \mathrm{~m}} \mathrm{Ba}$ is attenuated rapidly, the technique based on Equation 2 is limited to a few millimetres depth for ${ }^{137} \mathrm{Cs}$; the Compton technique potentially has a much greater depth range, to tens or hundreds of millimetres, and is not limited to nuclides with particular spectral characteristics.

\section{The influence of material type}

A key challenge in the research of non-intrusive, non-destructive techniques for depth assay of radioactive contamination is to account for the variety of materials in which radioactivity can be dispersed. There is, for example, a diverse range of construction materials in use across the generations of nuclear plant that now face decommissioning, and rarely does a complete historical record exist associated with, for example, the composition of concrete variants used in nuclear fuel storage ponds. Widening 


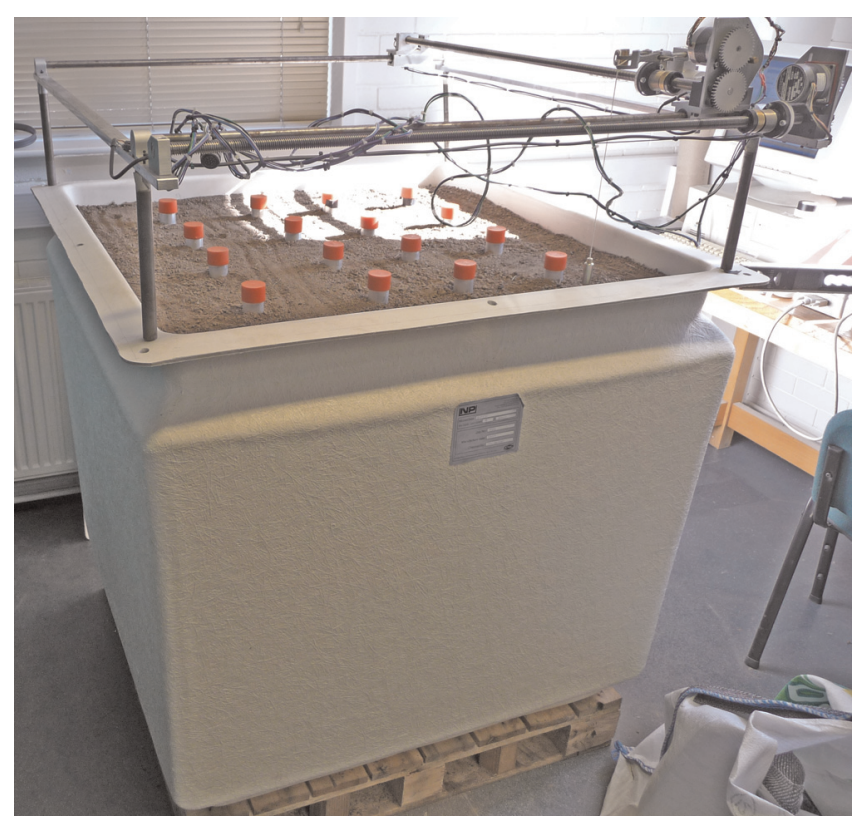

Figure 6. The large-scale phantom developed for contaminated land depth profiling research (Adams et al., 2010)

the potential of depth profiling techniques to the natural environments prone to contamination, engineers are required to address contaminated land and marine environments. For this purpose, a further dedicated phantom has been developed (Adams et al., 2010), which is shown in Figure 6. This comprises a $1 \mathrm{~m}^{3}$ vessel containing the medium under investigation (in Figure 5 it is shown containing dry, sterilised soil) together with 16 aluminium sample tubes to enable sealed sources to be placed at any depth in the phantom, simply and easily. A $\gamma$-ray detector can be placed at any position in the $x-y$ dimension above the tank to replicate land monitoring, and this is automated by means of the motorised scanning rig arranged above the phantom.

In addition to the variety of building materials and their composite aggregates, the ground also exhibits considerable variety. However, because the two most significant dependencies of photon attenuation are the atomic number and photon energy, and because almost all materials constituting construction materials and naturally-occurring terrain are generally limited to a relatively narrow range of $Z$, the specific material does not influence the measurement very significantly. This is demonstrated by the data in Figure 7, arising from a campaign of measurements with five contrasting media: sand, dry soil, wet soil, water and loose aggregate. These data indicate that the PCA technique is robust against variation in a wide range of silica-based terrestrial materials. It also indicates that repeated calibration with poorly understood, heterogeneous materials need not be necessary. This is particularly beneficial when legacy facilities are concerned because replicating the composition of the materials found in these facilities in the laboratory is unlikely to be straightforward.

\section{Example applications}

There are at least two possible applications of this technology. Radioactive particles in beach sediments and soils have been found in a number of places: Dounreay (SEPA, 2012a) and Sellafield (EA, 2009) resulting from nuclear-fuel reprocessing activities; and in several locations, including Dalgety Bay in

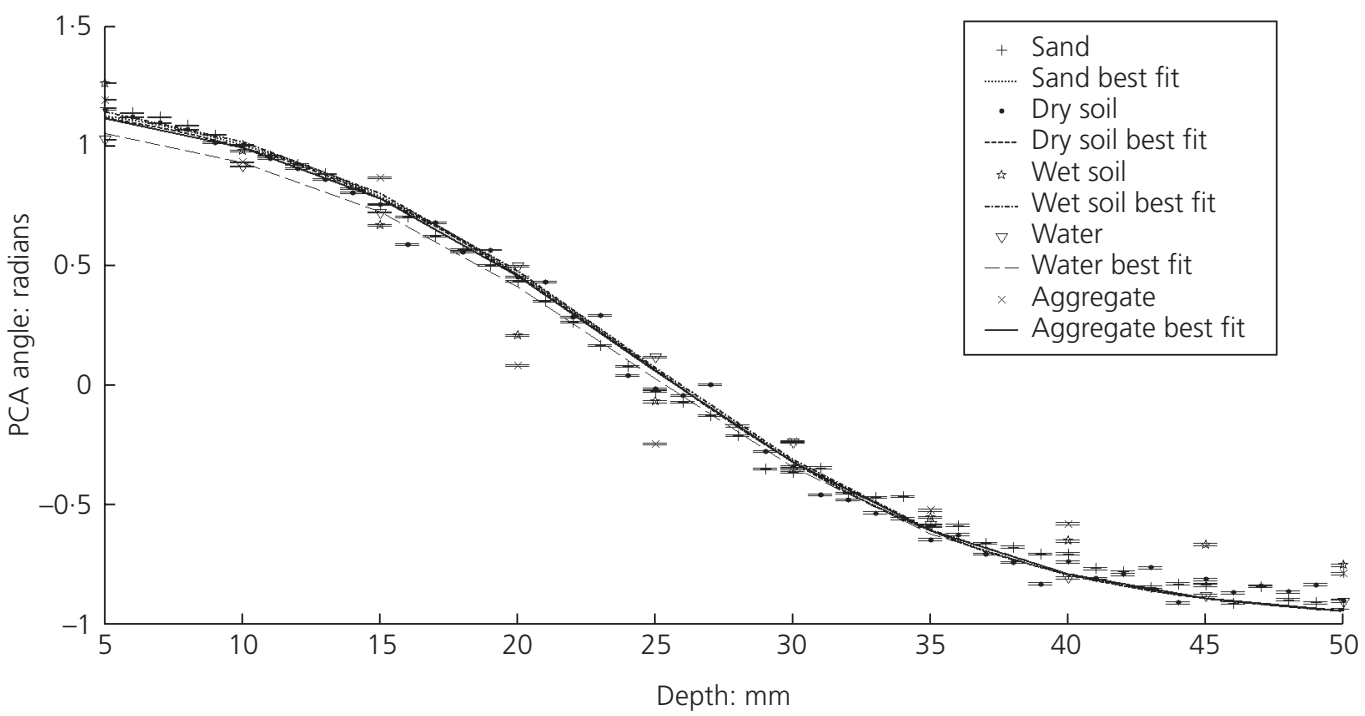

Figure 7. Principal component analysis (PCA) angle versus depth for caesium-137 in a variety of materials including sand, dry soil, wet soil, water and aggregate (Adams et al., 2012) 
Fife (SEPA, 2012b), where the radionuclide is a radium isotope $\left({ }^{226} \mathrm{Ra}\right)$, used in luminous dials for military and some domestic instruments. Given the widespread use of radium-based luminous paints in the last century, this problem is potentially widespread. ${ }^{226} \mathrm{Ra}$ produces a prominent $\gamma$-ray emission at $286 \mathrm{keV}$ and is therefore suitable for the technique presented here. The nature of these materials is that they are essentially point sources, directly analogous to the experimental data presented here. The recovery of a particle is potentially quicker and easier with less waste generation if a previous indication of its depth beneath the surface is made available.

Radioactive contamination is also found within solid concrete structures, where it has either diffused through mass concrete, or has penetrated along construction joints. The recovery of contamination from within mass concrete is much less easy than from soft sediments, and it is also potentially less of a long-term hazard. It may be that the best option is to recover the contaminated concrete or it may be that a case can be made that it is consistent with site de-licensing criteria (HSE, 2008 ) to leave it in place. In order to plan the work to recover the activity, or to make the decision to leave it in place, it is important to have information on the activity of contamination and its depth of burial. The potential advantage of the technique presented here is clear, although in this case the problem is more complex because the contamination may no longer be a point source of radiation. Further development in this area is under consideration.

\section{Future work}

The techniques summarised in this paper have been perfected in laboratory environments for a variety of media that constitute the majority of construction materials and ground that is open to inadvertent contamination on nuclear sites. However, this research has, to date, been limited to sealed, point-like sources that perhaps most effectively correspond to the scenario in which insoluble fragments of nuclear material constitute the contamination hazard.

Much of the contamination to be characterised, however, arises as a result of leaks and spills of radioactive liquid media that often results in dispersed contamination profiles both across the surface of materials and also varying with depth. A significant challenge of future research is to derive these profiles, especially for the case in which the composition of the radioactivity is poorly understood.

This paper has focussed on the proof of the techniques for ${ }^{137} \mathrm{Cs}$, and in the accompanying references ${ }^{60} \mathrm{Co}$ has also been investigated. Much contamination on decommissioning sites is associated with plutonium, which has the added complication that the characteristic $\gamma$-ray emissions are of much lower energies than is the case for ${ }^{137} \mathrm{Cs}$ or ${ }^{60} \mathrm{Co}$. This presents a challenge in terms of the maximum activity that is detectable at a given depth, or, conversely, the time needed for sufficient data to be accumulated and limits the extent to which the technique can be applied to plutonium contamination.

In future research, it is hoped that a variety of in-field measurements will be made on decommissioning sites to support the transfer of the technique from the laboratory to the sites that most need this characterisation capability. This will also require research to be carried out to identify the effect of voids on the response of the technique and the effect of high- $Z$ components in the media under study such as heavy metal components and rebar structures.

\section{Acknowledgements}

The authors would like to acknowledge the support of the Engineering and Physical Sciences Research Council (EPSRC), the Nuclear Decommissioning Authority (NDA), REACT Engineering Ltd, and the contributions of Jamie C. Adams and B. Alan Shippen.

\section{REFERENCES}

Adams JC, Joyce M and Mellor M (2010) A phantom for research studies of radiologically contaminated land. Paper N43-5. IEEE Nuclear Science Symposium, Oak Ridge, USA, November 2010, doi: 10.1109/NSSMIC.2010.5873965.

Adams JC, Mellor M and Joyce MJ (2011) The determination of the depth of localized radioactive contamination by ${ }^{137} \mathrm{Cs}$ and ${ }^{60} \mathrm{Co}$ in sand with principal component analysis. Environmental Science \& Technology 45(19): 8262-8267.

Adams JC, Joyce MJ and Mellor M (2012) Depth profiling ${ }^{137} \mathrm{Cs}$ and ${ }^{60} \mathrm{Co}$ non-intrusively for a suite of industrial shielding materials and at depths beyond $50 \mathrm{~mm}$. Applied Radiation and Isotopes 70(7): 1150-1153.

Cruickshank J (2012) The Sellafield Contaminated Land and Groundwater Management Project: Characterisation of a Complex Nuclear Facility. Workshop on Radiological Contamination for Decommissioning, Nyköping, Sweden. See http://oecd-nea.org/rwm/wpdd/rcd-workshop/C-2_OH_ The_Sellafield_contaminated_land_and_groundwater.pdf. pdf (accessed 15/02/2013).

EA (Environment Agency) (2009) Sellafield radioactive particles in the environment: strategy for responding to particle finds. See http://www.environment-agency.gov.uk/ static/documents/Business/EA_Strategy_for_Responding_ to_Particle_Finds.pdf (accessed 15/02/2013).

Fourie E (2012) NECSA South Africa Environmental Protection. See http://www.iaea.org/OurWork/ST/NE/ NEFW/WTS-Networks/ENVIRONET/environetfiles/ ENV_AnnFor_presentations/Overview_SouthAfricaFOURIE.pdf (accessed 15/02/2013).

HSE (Health and Safety Executive) (2008) Guidance to inspectors on the interpretation and implementation of the 
HSE policy criterion of No Danger for the delicensing of nuclear sites. See http://www.hse.gov.uk/nuclear/ delicenceguide.pdf (accessed 15/02/2013).

Mahe C (2011) Characterisation and visualisation technologies in $D D \& R$. See http://www.iaea.org/OurWork/ST/NE/ NEFW/WTS-Networks/IDN/idnfiles/ Characterization\&Visualization/06-Gamma_Spectrometry. pdf (accessed 15/02/2013).

SEPA (Scottish Environment Protection Agency) (2012a) Dounreay particles research. See http://www.sepa.org.uk/ radioactive_substances/decommissioning/dounreay/ dounreay_particles_research.aspx (accessed 31/12/2012).
SEPA (2012b) Dalgety Bay. See http://www.sepa.org.uk/ radioactive_substances/dalgety_bay.aspx (accessed 31/12/2012).

Shippen BA and Joyce MJ (2010) Profiling the depth of caesium-137 contamination in concrete via a relative linear attenuation model. Applied Radiation and Isotopes 68(4-5): 631-634.

Shippen BA and Joyce MJ (2011) The design and calibration of a phantom for depth profiling measurements of entrained radioactivity in silica-based media. Nuclear Engineering and Design 241(2): 526-532.

\section{WHAT DO YOU THINK?}

To discuss this paper, please email up to 500 words to the editor at journals@ice.org.uk. Your contribution will be forwarded to the author(s) for a reply and, if considered appropriate by the editorial panel, will be published as a discussion in a future issue of the journal.

Proceedings journals rely entirely on contributions sent in by civil engineering professionals, academics and students. Papers should be 2000-5000 words long (briefing papers should be 1000-2000 words long), with adequate illustrations and references. You can submit your paper online via www.icevirtuallibrary.com/content/journals, where you will also find detailed author guidelines. 